\title{
Cerebellar syndrome in myxoedema revisited: a published case with carcinomatosis and multiple system atrophy at necropsy
}

\author{
N Quinn, R O Barnard, R E Kelly
}

\begin{abstract}
One of six patients in a 1960 paper on "Cerebellar syndrome in myxoedema" was subsequently found to have adenocarcinoma. General post-mortem revealed carcinomatosis and basal pneumonia. Neuropathological examination revealed the changes of multiple system atrophy. The relationship between hypothyroidism, carcinoma, and cerebellar, pontine and striatonigral degeneration is discussed.
\end{abstract}

In 1960 Jellinek and Kelly ${ }^{1}$ described six cases of cerebellar syndrome in myxoedema. We report the clinical history, subsequent course and pathological findings of their case six.

\section{Case report}

From the age of 60 , this patient gradually developed dysarthria and ataxia. There was no alcohol abuse, and no family history of neurological illness. Although hypothyroidism was not suspected clinically, investigation fourteen months later showed serum cholesterol was elevated (400 mg\%, $10.4 \mathrm{~mol} / \mathrm{l}$ ) BMR was $-10 \%$ and $I^{131}$ uptake was just below the lower limit of normal. Because his physician (REK) had recently established a link between hypothyroidism and a cerebellar syndrome, a tentative diagnosis of cerebellar degeneration secondary to hypothyroidism was made, and he was started on thyroid supplements. His gait improved considerably, but not his dysarthria.

However, his clinical state then deteriorated further. After a further 15 months he was severely dysarthric, with gait and limb ataxia and also cogwheel rigidity in the right arm, without tremor. $\mathrm{He}$ fell and fractured his femur, and when reassessed his tendon reflexes were also increased on the right side and both plantar responses were extensor. Cholesterol and BMR (on treatment) were normal.

His movements became progressively slower, and by the time of his final admission at the age of 65 years he could no longer walk. Micturition and bowel function were normal, but no specific enquiry about potency or postural faintness was made. His speech was almost unintelligible. Despite this, his intellect appeared intact. Finger movements were markedly slowed, there was a slight action tremor, and cogwheel rigidity was present in all four limbs. There was slight heel-shin ataxia, and slight generalised wasting of both legs, with fasciculations evident in both calves. The reflexes were normal or depressed, but both plantar responses were extensor. Sensation was intact apart from impaired vibration sense at the ankles. The liver was enlarged two to three fingerbreadths to percussion. Alkaline phosphatase and ESR were elevated. A liver biopsy showed deposits of adenocarcinoma. The diagnosis was changed to carcinomatous cerebellar degeneration. The patient was then transferred for terminal care to his local hospital. He died one month later, four and a half years after the onset of his neurological symptoms.

\section{Necropsy examination}

There was a well-differentiated adenocarcinoma of the stomach with metastatic deposits in the lymphatics, lymph nodes, liver and lung. A left basal pneumonia was present. The thyroid was of normal size and texture.

The brain weighed $1215 \mathrm{gm}$ complete; the cerebellum and brainstem weighed $125 \mathrm{gm}$. There was moderate generalised atrophy of cerebral convolutions, with mild leptomeningeal thickening; the cerebellum and brain stem were conspicuously small, but symmetrical. Coronal slices made after formalin fixation disclosed no changes in the cerebral cortex or white matter, but notable symmetrical atrophy of the central grey matter. The heads of the caudate nuclei were shrunken, and the ventricles symmetrically enlarged. The putamen and globus pallidus were shrunken, the former showing marked dark brown discolouration. The thalami and subthalamic nuclei were also atrophic. The whole brain stem was abnormally small, with dilatation of the aqueduct and fourth ventricle. The substantia nigra was uniformly very pale due to severe loss of pigment, but the red nuclei were preserved. The basis pontis was very atrophic. All cerebellar peduncles were very small. The medulla was atrophied and the inferior olives were difficult to recognise. The cerebellum showed generalised atrophy and obvious gliosis and the dentate nucleus was tiny and had lost its normal convolutional pattern. The spinal cord was of normal appearance.

The thyroid gland was normal as were the cerebral cortex and white matter. In the caudate, putamen and globus pallidus there was marked neuronal loss and astrocytic proliferation. The dark finely granular pigment in the putamen, partly taken up by phagocytes, 
was iron. In all the thalamic nuclei loss of neurons and glial proliferation were seen. Very few normal pigmented neurons remained in any part of the substantia nigra or in locus coeruleus. Melanin was present as free-lying pigment or within phagocytes and there was gliosis. No Lewy bodies or neurofibrillary changes were found. In the atrophied pons the loss was especially marked in the transverse pontine fibres and the pyramidal fibres were relatively spared. Reduction in neurons was conspicuous in the atrophied inferior olives. In the cerebellum focal loss of granule cells was severe, loss of Purkinje cells was accompanied by "torpedos" and some "empty baskets". Some atrophic Purkinje cells were displaced into the molecular layer, where isomorphic gliosis was common. The population of the dentate nucleus was poor and the cerebellar white matter was gliotic.

In the spinal cord there was loss of myelinated fibres in the lateral pyramidal tract and in posterior columns with gliosis. In the cervical cord there was some loss of anterior horn cells and gliosis. In the thoracic cord Clarke's column was normal. The intermedio-lateral column was normal. Posterior root ganglia showed non-specific changes of patchy mild neuronal loss with capsule cell proliferation. Perivascular lymphocytic cuffing was not found in the cord or ganglia.

\section{Discussion}

When this patient was initially investigated for a cerebellar syndrome, hypothyroidism was found on investigation. This association, together with the clinical improvement with thyroid supplementation, was in keeping with the five other cases of cerebellar syndrome with myxoedema reported in the same paper. ${ }^{1}$ In the absence of any necropsy data, the authors had proposed unknown metabolic or vascular processes which may lead to degeneration, yet may still respond to treatment. They contrasted this total or partial remission with the subacute cerebellar degeneration of malignant disease $^{2}$ which did not remit. Pathological material from patients with an apparently isolated myxoedematous cerebellar syndrome is scant. In 1966 Price and Netsky ${ }^{3}$ described cerebellar Purkinje cell loss in a man with ataxia and myxoedema, but he had also been an alcoholic. Subsequently in 1971 Barnard et $a l^{4}$ described pathological findings in a 57 year old woman with severe hypothyroidism and a marked cerebellar syndrome. There was cerebellar degeneration but also pontine atrophy. Review of material from this case suggests the possibility of MSA.

In 1951, Brain et $a l^{2}$ had described four cases of subacute cortical cerebellar degeneration, and stressed the relation to carcinoma in three of them. Death occurred between three and seven months from the onset of cerebellar symptoms. Pathologically, there was widespread and diffuse degeneration and disappearance of Purkinje cells, with only minor changes in the dentate nucleus. The brainstem, basal ganglia and cerebral cortex were essen- tially spared, and the olives were usually normal. In the three patients with carcinoma, the CSF showed a lymphocytic pleocytosis, and in the two in which it was examined the spinal cord showed perivascular lymphocytic cuffing. Today, these patients could be considered to have encephalomyelitis with carcinoma ${ }^{5}$ as opposed to the more common noninflammatory restricted cortical cerebellar degeneration associated with malignancy. ${ }^{6}$

In 1965, Brain and Wilkinson ${ }^{7}$ added three further cases of subacute cerebellar degeneration. Their case 2 is striking for its atypical clinical and pathological picture. This man developed a cerebellar syndrome at the age of 39. Eleven months later he had additional parkinsonism with a pill-rolling tremor, but no pyramidal signs. He developed jaundice, and died of liver failure 15 months after the onset of his illness. Necropsy examination revealed a peripheral carcinoma of the lung with secondary deposits in the liver and right adrenal. The striatum was normal, but the globus pallidus showed marked pallor in preparations stained for myelin, with some loss of nerve cells. The substantia nigra, pons and medulla were normal. The cerebellar cortex showed severe loss of Purkinje cells, but no evidence of an inflammatory process. The pathological conclusions were: 1) cerebellar atrophy of Purkinje cell type and 2) probable pallidal degeneration.

In 1989 Golbe et $a l^{8}$ described rapidly progressive dystonia-Parkinsonism in a 42 year old woman with breast carcinoma. Interestingly, she also had a 10 year history of hypothyroidism, treated with thyroxine. She developed intermittent tremor and loss of dexterity in both hands, followed by difficulty with her balance. Marked weight loss prompted systemic investigation, which revealed breast adenocarcinoma with hepatic secondaries. CT brain scan was normal. The movement disorder progressed rapidly: she developed facial hypomimia, severe hypophonic dysarthria, dysphagia, moderate bradykinesia, rigidity, and dystonic posturing, with a coarse 4-6 Hz action tremor, of both arms, but no pyramidal signs. Treatment with levodopa was unhelpful, and the patient died of aspiration pneumonia four months after the onset of neurological symptoms. At necropsy, the brain showed mild astrocytosis in each outer globus pallidus. There was mild to moderate loss of nigral neurons, worse in the pars reticulata, and mild gliosis. Sections of pons and medulla were unremarkable. The cerebellum showed mild loss of Purkinje cells, with minimal Bergmann gliosis. There were no Lewy bodies, and neither tumour nor inflammatory cells were present.

These two young cases with neoplasia developing a rapidly progressive and fatal neurological picture, including Parkinsonism, raise the possibility that a paraneoplastic syndrome might rarely manifest as an extrapyramidal disorder.

Could our case of apparent MSA be entirely explained as a paraneoplastic syndrome? The longer neurological symptom duration of 4 and 
a half years, together with the age of the patient and the combination of sites involved, would be more in keeping with "classical" cases of MSA. Moreover, gastric cancer rarely causes a cerebellar paraneoplastic syndrome.

Among cases of pathologically proven MSA, median age at death is around 59 years. Occasional subjects with MSA and coincidental malignancy might therefore be expected, but we have found only one. Case 3 of Adams $e t a^{10}$ (case 4 in their 1964 paper $^{11}$ ) developed Parkinsonism at the age of 51.Three years later she had brisk reflexes, flexor plantar responses, ataxia and intention tremor. The discovery of an abdominal mass led to a laparotomy, which revealed ovarian adenocarcinoma. She died 28 hours later. Necropsy showed cell loss and gliosis in putamen, caudate, globus pallidus, substantia nigra, pontine nuclei and olives, with moderate loss of cerebellar Purkinje cells. There was no evidence of perivascular inflammation.

On balance, we feel that our patient had MSA together with incidental gastric carcinoma and possible hypothyroidism. Whilst hypothyroidism was clearly not the sole cause of the patient's initial cerebellar syndrome, the fact that he improved, albeit only partially and temporarily, on replacement therapy suggests that, as well as causing cerebellar symptoms in some patients, hypothyroidism might worsen function in patients with other cerebellar degenerations.

1 Jellinek EH, Kelly RE. Cerebellar syndrome in myxoedema. Lancet 1960;ii:225-7.

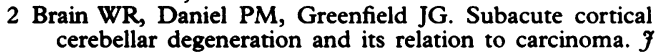
Neurol Neurosurg Psychiatry 1951;14:59-75.

3 Price TR, Netsky MG. Myxoedema and ataxia. Cerebellar alterations and "neural myxoedema bodies". Neurology 1966;16:957-62.

4 Barnard RO, Campbell MJ, McDonald WI. Pathological findings in a case of hypothyroidism with ataxia. $\mathcal{F}$ Neurol Neurosurg Psychiatry 1971;34:755-60.

5 Henson RA, Hoffman HL, Urich H. Encephalomyelitis with carcinoma. Brain 1965;88:449-64.

6 Henson RA, Urich H. Cancer and the nervous system. The neurological manifestations of systemic malignant disease. Oxford: Blackwell, 1982;314-67.

7 Brain WR, Wilkinson M. Subacute cerebellar degeneration associated with neoplasms. Brain 1965;88:465-78.

8 Golbe LI, Miller DC, Duvoisin RC. Paraneoplastic degeneration of the substantia nigra with dystonia and parkinsonism. Movement Disorders 1989;4:147-52.

9 Quinn N. Multiple system atrophy. In: Marsden CD, Fahn S, eds. BIMR vol 12: Movement disorders 3. London: Butterworths (In press)

10 Adams R, van Bogaert L, van der Eecken H. Dégénérescences nigro-striées et cérébello-nigro-striées. Psychiat Neurol 1961;142:219-59.

11 Adams RD, van Bogaert L, van der Eecken H. Striato-nigral degeneration. $\mathcal{f}$ Neuropathol Exp Neurol 1964;23: 584-608. 